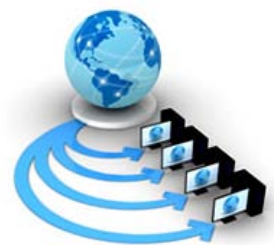

Volume 9, No. 2, March-April 2018

International Journal of Advanced Research in Computer Science

RESEARCH PAPER

Available Online at www.ijarcs.info

\title{
MULTIDIMENSIONAL ANALYSIS OF GENETIC ALGORITHM USING MATLAB
}

\author{
Prof. Neeraj Bhargava \\ School of System Science \& Engineering \\ MDS University Ajmer, India
}

\author{
Dr. Ritu Bhargava \\ Lecturer, Sophia Girls’ College \\ Ajmer, India
}

\author{
Kapil Chauhan \\ Aryabhatt College \\ Ajmer, India
}

\begin{abstract}
As the utilization of frameworks are expanding in different parts of our everyday life, it improves the complexity of frameworks in Software outline and equipment segments (caches).Within the recent years the Test Engineers have built up another testing strategy for testing the accuracy of frameworks: to be specific the developmental test. The test is deciphered as an issue of improvement, and utilizes developmental calculation to discover the test information with extraordinary execution times. Developmental testing indicates the utilization of transformative algorithms, e.g., Genetic Algorithms, to help different test computerization undertakings. Since developmental algorithms are heuristics, their execution and yield productivity can differ over various runs, there is solid need a domain that can be handle these complexities, MATLAB is generally utilized for this reason. This paper investigate Genetic algorithm the energy of Genetic Algorithm for advancement by utilizing MATLAB in view of usage of Rastrigin's capacity, in this paper we utilize this capacity as streamlining issue to clarify some key meanings of genetic change like determination Optimal fitness, mutation, sore, selection.
\end{abstract}

Keywords: Evolutionary Testing, Genetic Algorithm, Fitness, Selection, Mutation, MatLab

\section{INTRODUCTION}

Genetic algorithms are a way to deal with learning construct freely in light of standards of natural development, these are easy to build, and its execution does not require a lot of capacity, settling on them an adequate decision for an enhancement issues. Ideal planning is a nonlinear issue that can't be unraveled effectively yet, a GENETIC ALGORITHM could serve to locate a respectable arrangement in a restricted measure of time Genetic algorithms are roused by the Darwin's hypothesis about the advancement "survival of fittest", it look through the arrangement space of a capacity using recreated development (survival of the fittest) system. By and large the fittest people of any populace have more prominent opportunity to replicate and make due, to the cutting edge accordingly it add to enhancing progressive ages However second rate people can by chance survive and furthermore repeat, Genetic algorithms have been appeared to take care of straight and nonlinear issues by investing Genetic algorithm all districts of the state space and exponentially abusing promising territories through the use of transformation, hybrid and choice activities to people in the populace. The improvement of new software innovation and the new software situations (e.g. MATLAB) give the stage to taking care of troublesome issues progressively. It coordinates numerical examination, network calculation and illustrations in a simple to utilize environment.[1]

MATLAB capacities are straightforward content records of translated guidelines Therefore; these capacities can be reactualized starting with one equipment design then onto the next without even a recompilation step. MATLAB (Matrix Laboratory), a result of Math works, it is a logical software bundle created to give a coordinated domain to numeric calculation and illustrations perception in abnormal state programming dialect.[2]

MATLAB has a wide Genetic algorithm of capacities valuable to the genetic algorithm expert and those wishing to explore different avenues Genetic algorithm the genetic algorithm. In MATLAB's abnormal state dialect, issues can be coded in m-records in a small amount of the time that it would take to make C or FORTRAN programs for a similar reason. It likewise gives propelled information investigate Genetic algorithm, perception apparatuses and unique reason application space toolboxes. This paper is sorted out into three sections: In segment I portray the helpfulness of GENETIC ALGORITHM and highlights of new software MATLAB. In segment II examines the execution issues of GENETIC ALGORITHM in different accessible dialects, apparatuses and software. At last GENETIC ALGORITHMs is actualized utilizing MATLAB for the Rastrigin's capacity as contextual Genetic algorithm for advancement. In segment III finishes up the destinations of paper [3].

\section{INTRODUCTION OF MATLAB TO IMPLEMENTATION OF GENETIC ALGORITHM}

The execution of genetic algorithm on superior PCs is a troublesome and tedious undertaking. The executing dialects must be nearly as conceivable to the scientific portrayal of the issue, basic and simple to-utilize procedural dialect. The $\mathrm{C} / \mathrm{C}++$, FORTRAN are bring down level assembled programming dialects (in some cases named a third era dialect) that is generally utilized as a part of the scholarly community, industry, business and GENETIC ALGORITHM is likewise actualized by utilizing these classification of dialects. The primary preferred standpoint of accumulated low-level dialects is their execution speed and 
proficiency but now days the MATLAB is often utilized in research and industry and it is a case of an abnormal state "scripting" or "fourth era" dialect. The most conspicuous contrast between ordered dialects and deciphered dialects is that the mediator program peruses the source code and makes an interpretation of it into machine directions on the fly, i.e. no Genetic algorithm is required. This reductions the execution speed however it make the software engineer free from memory administration, permits dynamic writing and intuitive sessions. [4]

Note that the projects sent in scripting dialects are generally altogether shorter than proportionate projects

Written in ordered dialects and furthermore set aside essentially less opportunity to code and troubleshoot. To put time and the advancement time. The client can type one or a few orders at the charge quick and subsequent to squeezing return, these orders are executed promptly. By this it permits the software engineer for intuitive testing of little parts of the code (immediately originating from Genetic algorithm and empowers experimentation) [5]. The MATLAB bundle accompanies modern libraries for grid activities, general numeric strategies and plotting of information, in this manner MATLAB turn out to be first decision of software engineer to actualize logical, graphical and scientific applications and for the GENETIC ALGORITHM usage MATLAB is accompanied unique apparatus that is Genetic Algorithmdevice.[6]

\section{IMPLEMENTATION GENETIC ALGORITHMS}

In fact a GENETIC ALGORITHM is to choose on the off chance that it is conceivable to naturally fabricate arrangements on issue. For instance, in the Traveling Salesman Problem, each course that goes through the urban communities being referred to is conceivably an answer, albeit most likely not the ideal one. It is fundamental that in light of the fact that a GENETIC ALGORITHM requires an underlying populace $\mathrm{P}$ of arrangements.

At that point choose what "quality" portrayal will utilize we have a couple of choices like twofold, whole number, twofold, stage, and so forth. The paired and twofold being the most normally utilized since they are the most adaptable. In the wake of choosing the quality portrayal it must be choose: The strategy to choose guardians from the populace $\mathrm{P}$, the way these guardians will "mate" to make relatives, the change technique (discretionary however valuable), and the strategy will use to populate the people to come and the calculation's end condition (number of ages, time constrain, worthy quality edge). Presently second thing is Processor and working framework that must be fit for running the program the calculation is coded in MATLAB. Matlab gives an improvement tool kit that incorporates a GENETIC ALGORITHM-based solver. [7] The apparatus compartment can be starting by composing gatool in the Matlab. At the point when the change window shows up, we can pick the solver Genetic algorithm - Genetic Algorithm and now matlab are set up to go. The customer should program any extended value required. We will complete Rastrigin's Functions in the most ideal field and number of variable is 2 and populace create is twofold vector. (Figure 1).[8]

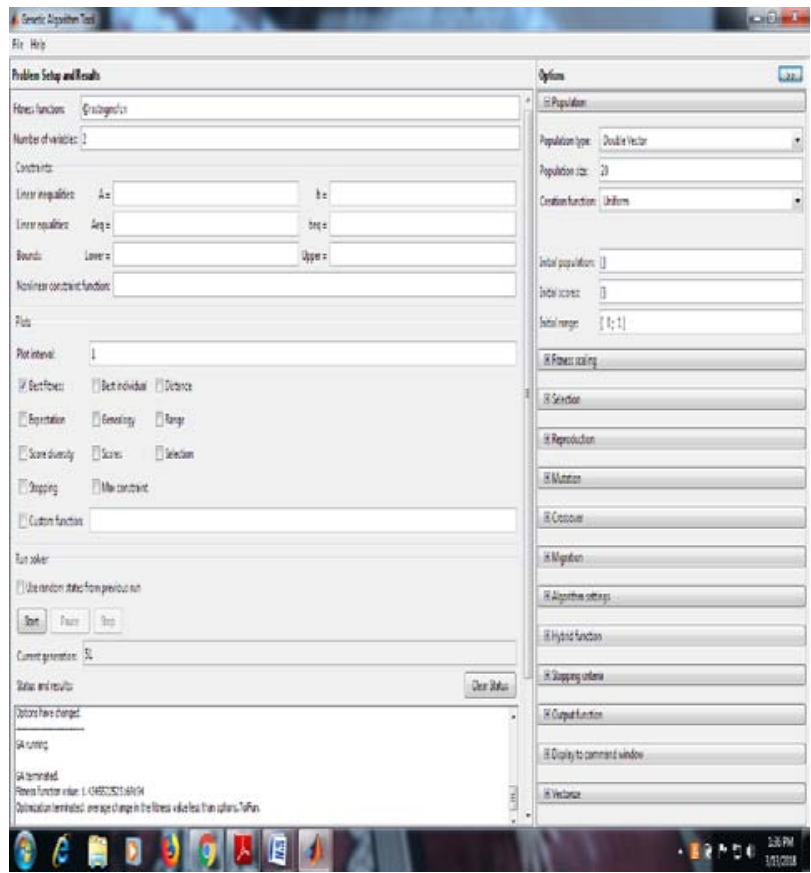

Figure:1 Genetic Algorithm in Matlab's Optimization Toolbox

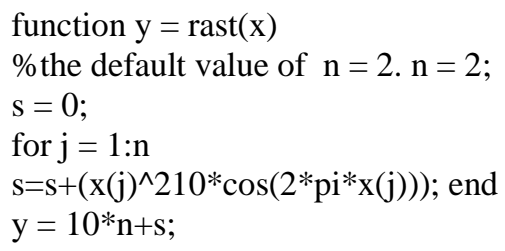

The ideal fitness function value esteem and the end condition met are printed, together with the plan. Since the strategy is stochastic, don't would like to have the ability to copy any result found in a substitute run. By and by check the two plots on the left. Plainly, the masses join together, since the typical partition between individuals (game plans) in term of the health regard is decreased, as the ages pass. This is a measure of the fair assortment of a people. It is hard to avoid meeting yet keeping it low or deferring its appearance is better. Having grouped assortment in the masses empowers the GENETIC ALGORITHM to look for better in the course of action space. [9]

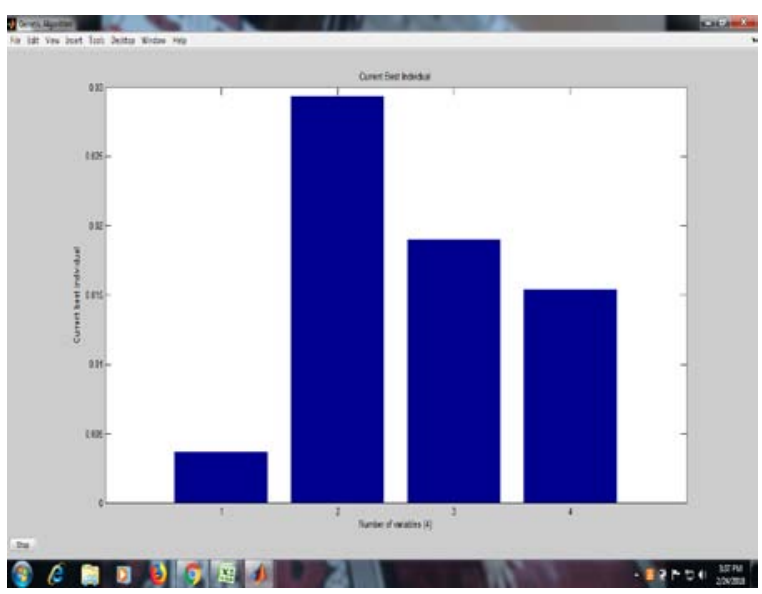

Figure: 2 Optimal Individual In Genetic Algorithm 
It is in figure-2 the optimal individual incentive as it bit by bit gets littler. It means that advancement happens since not just the wellness estimation of the optimal individual was diminished, even the mean (normal) wellness of the population was additionally reduced.[10]

a. Population Diversity- the execution of a GENETIC ALGORITHM is influenced by the diversity of the underlying population. On the off chance that the normal separation between people is expansive, it is sign of high diversity; if the normal separation is little its speak to low diversity in the population.

In the event that the diversity is too high or too low, the hereditary calculation won't not perform well. We will clarify this by the accompanying: By default, the Optimization Tool makes an irregular introductory population utilizing a creation work. We can restrict this by setting the Initial range field in Population choices. By this we really make it harder for the GENETIC ALGORITHM to seek similarly well in every one of the arrangements space. This will permit the calculation keep running for 100 age giving us better outcomes (and plots).The GENETIC ALGORITHM restores the optimal wellness work estimation of 2 .

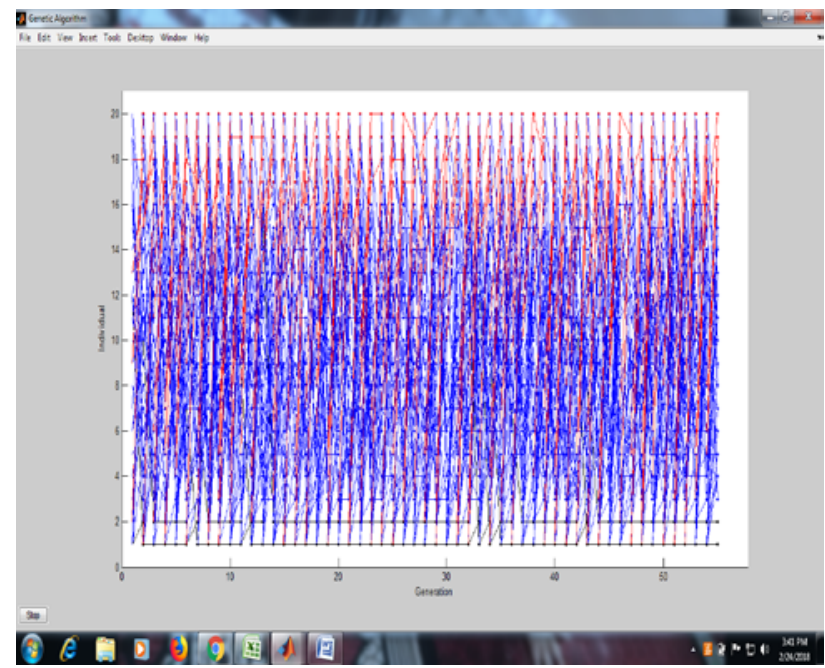

Figure 3: Genealogy in Genetic Algorithm

The upper plot, which shows the optimal wellness at every age, demonstrates little advance in bringing down the wellness esteem (dark specks) in fig 4. The lower plot demonstrates the normal separation between people at every age, which is a decent measure of the assorted variety of a populace. For this setting of beginning extent, there is too little assorted variety for the calculation to Genetic algorithm ground. The calculation was caught in a neighborhood least because of the underlying reach limitation.

Next, set Initial range to $[1 ; 60]$ and run the calculation once more. The GENETIC ALGORITHM restores the optimal wellness estimation approximately 3.7. This time, the hereditary calculation Genetic algorithm ground, but since the normal separation between people is so extensive, the optimal people are a long way from the ideal arrangement.

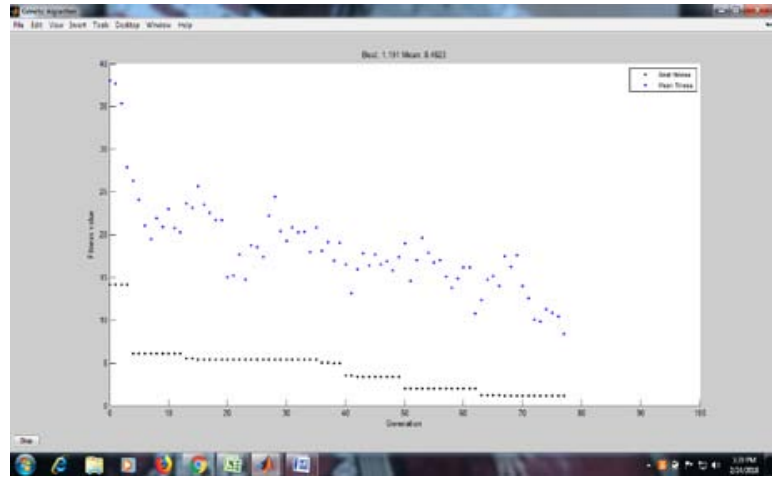

Figure 4: fitness in Genetic Algorithm

Set Initial range to $[2 ; 3]$ and run the GENETIC ALGORITHM. This returns the optimal fitness value of approximately 0.023 and displays the plots that follow as in figure 4

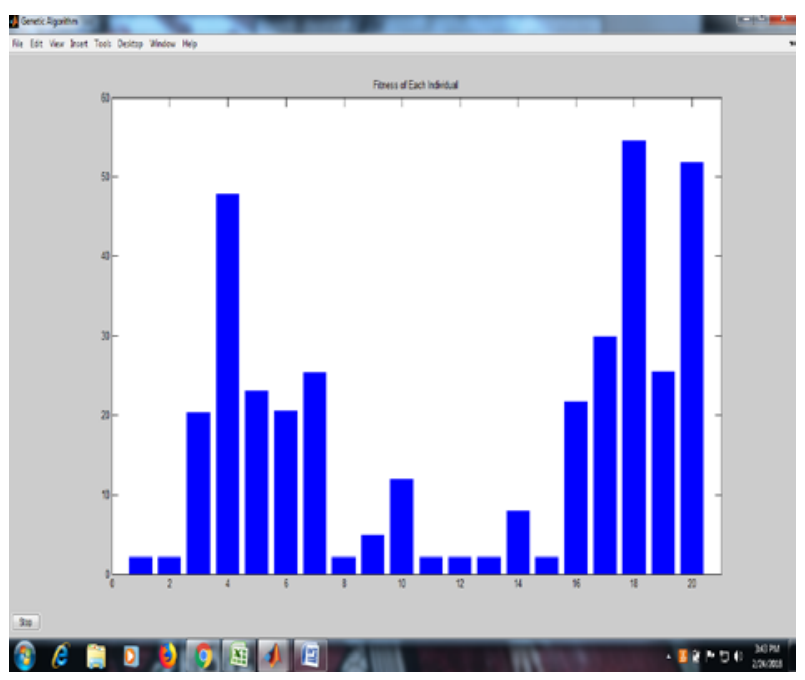

Figure 5: score in Genetic Algorithm

The varying assortment for this circumstance is more fit the bill to the issue, so the inherited figuring reestablishes a tremendously enhanced result than in the previous two cases.

This Genetic algorithm chooses the degree of the populace at each age. Extending the populace Genetic algorithm enables the inherited count to look more concentrations and thus procure a predominant result. In any case, the greater the populace measure, the more drawn out the inherited estimation takes to figure each age. [11]

Note that Population Size to be at any rate the estimation of Number of elements, so the general population in each people cross the space being looked.

Finally, another parameter that impacts the fair assortment of the people is the Fitness Scaling. If the health Genetic algorithm move too for the most part Figure: 4, the general population with the most negligible Genetic algorithm (audit that we confine) rehash too rapidly, expecting control over the people pool too quickly and shielding the GENETIC ALGORITHM from looking for changed domains of the plan space. On the other hand, if the characteristics change only a little, all individuals have approximately a comparable plausibility of multiplication and the task will progress step by step.[12] 


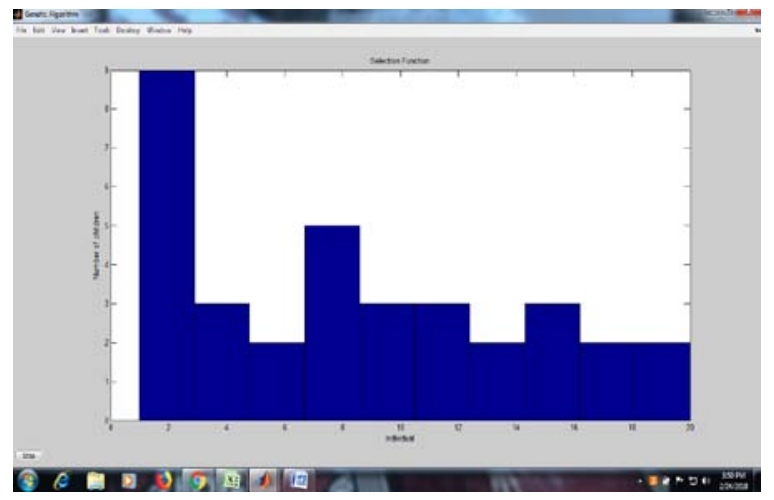

Figure 6: Selection in Genetic Algorithm

The Fitness Scaling modifies the wellness esteems (scaled esteems) previously the choice advance of the GENETIC ALGORITHM. This is managed without changing the positioning request, that is, the optimal individual in view of the crude wellness esteem remains the optimal in the scaled rank, too. Just the qualities are changed, and along these lines the likelihood of a person to get chose for mating by the determination system. This keeps the GENETIC ALGORITHM from joining too quick which enables the calculation to better hunt the arrangement space. We proceed with Rastrigin's capacity implantation in MATLAB. The Selection board in Options controls the Selection Function, that is, the means by which people are chosen to wind up guardians. Note that this system deals with the scaled esteems. Most outstanding techniques are displayed (uniform, roulette and competition). An individual can be chosen more than once as a parent, in which case it contributes its qualities to in excess of one child.[13]

The default assurance decision, Stochastic Uniform in which each parent analyzes to a zone of the line of length comparing to its scaled Genetic algorithm. For example, expect a populace of 4 individuals with scaled Genetic algorithm 7, 4, 3 and 1 . The individual with the scaled estimation of 7 is the optimal and should contribute its characteristics more than the rest. We make a line of length $1+3+4+7=15$. By and by, assume that we need to pick 6 individuals for Genetic algorithm. We wander over this line in endeavors of 15/6 and select the individual for cross breed.

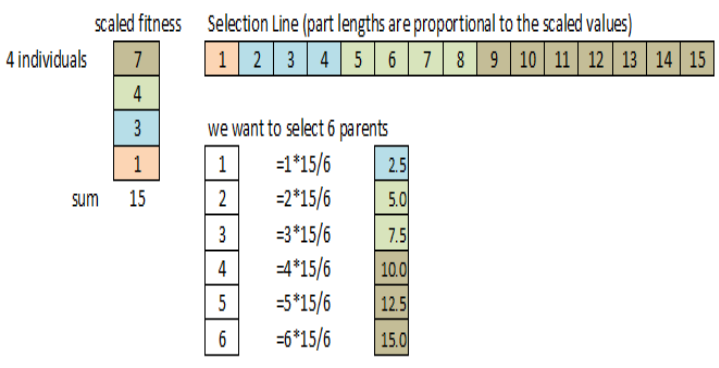

Figure 7: Stochastic selection method.

The Reproduction board in Options controls how the GENETIC ALGORITHM makes the general population to come. Here you show the measure of elitism and the division of the quantity of tenants in the bleeding edge that is made through mutation. The choices are: Elite Count: the amount of individuals with the optimal health Genetic algorithm in the present age that are guaranteed to get by to the general population to come.

a. Crossover Fraction: cross portion is the part of people in the people to come, other than first class kids, that are made by hybrid (remaining is produced by transformation). A hybrid portion of ' 1 ' demonstrates implies that all kids other than world class people are hybrid youngsters. A hybrid portion of ' 0 ' demonstrates that all youngsters are change kids. [14]

b. Two-point hybrid two hybrid focuses are chosen, double string from the earliest starting point of the chromosome to the principal hybrid point is replicated from the primary parent, the part from the first to the second hybrid point is duplicated from the other parent and the rest is replicated from the main parent once more.[14]

c. Mutation-It is the Random change at least one digits in the string speaking to an individual.[14].

\section{CONCLUSION}

The primary goal in this paper is that how the new innovation of MATLAB can be utilized as a part of request to execute a genetic algorithm in streamlining issues. It utilizes the energy of genetic algorithms to produce quick and effective arrangements continuously. The outcomes demonstrate that GENETIC ALGORITHM Tool can enhance wellness esteem by giving rapidly an arrangement of close ideal arrangements. Concerning the impact of various GENETIC ALGORITHM parameter setups, it found that an expansion in populace size can enhance execution of the framework. The parameter of hybrid rate does not influence the nature of the arrangement. Genetic Algorithms are anything but difficult to apply to an extensive variety of enhancement issues, similar to the inductive idea getting the hang of, booking, and design issues. The outcome demonstrates that the proposed GENETIC ALGORITHMs with the particular can discover arrangements with better quality in shorter time. The designer utilizes this data to look, find, and investigate Genetic algorithm the shortcomings that caused the disappointments. While every one of these territories for future thought could be additionally explored as for materialness for software testing, since it is likewise an improvement issue with the target that the endeavors devoured ought to be limited and the quantity of shortcomings recognized ought to be augmented. At long last, it would enthusiasm for additionally research to test a progression of various frameworks keeping in mind the end goal to see the relationship between's genetic algorithm and framework exhibitions.

\section{REFERENCES}

[1] D.E. Goldberg, Genetic Learning in optimization, search and machine learning. Addisson Wesley, 1994.

[2] J.J. Grefenstette. Genetic algorithms for changing environments. In R. Manner abd B. Manderick, editor, Parallel Problem Solving from Nature 2, pages 465-501. Elsevier Science Publishers.

[3] Goldberg, David E., and Kalyanmoy Deb. "A comparative analysis of selection schemes used in genetic algorithms." Urbana 51 (2015): 61801-2996.

[4] Henriksson, D., Cervin, A., Arzen, K.E.: TrueTime: Realtime control system simulation with MATLAB/Simulink. In: Proceedings of the Nordic MATLAB Conference, Copenhagen, Denmark (2015) 
[5] A.J. Chipperfield, P. J. Fleming and H. Pohlheim, "A Genetic Algorithm Toolbox for MATLAB," Proc. International Conference on Systems Engineering, Coventq, UK, 6-8 Sept 2016.

[6] Hoque, Mohammad Sazzadul, et al. "An implementation of intrusion detection system using genetic algorithm." arXiv preprint arXiv:1204.1336 (2014).

[7] K. Lakhotia, M. Harman, and P. McMinn. A multi-objective approach to search-based test data generation. In Proc. 9th Annual Conf. on Genetic and Evolutionary Computation (GECCO’07), pages 1098-1105, ACM, 2016

[8] Fogel, L. J., Owens, A. J. and Walsh, M. J.: Artificial Intelligence through Simulated Evolution. New York: John Wiley, 2015

[9] Cantú-Paz, E.: A Summary of Research on Parallel Genetic Algorithms. Technical Report IlliGENETIC ALGORITHML No. 95007, July 1995, University of Illinois at UrbanaChampaign, 1995
[10] Rishita Kalyani,” Application of Multi-Core Parallel Programming to a Combination of Ant Colony Optimization and Genetic Algorithm”, Indian Journal of Science and Technology, Vol 8(S2), 138-142, January 2015.

[11] Schöneburg, E., Heinzmann, F. and Feddersen, S.: Genetische Algorithmen und Evolutionsstrategien. Bonn, Paris, Reading, Mass.: Addison-Wesley, 2015.

[12] Grefenstette, J. J.: Proceedings of the Second International Conference on Genetic Algorithms and their Application, Hillsdale, New Jersey, USA: Lawrence Erlbaum Associates, 2014.

[13] Chipperfield, A. J., Fleming, P. J. and Pohlheim, H.: A Genetic Algorithm Toolbox for MATLAB. Proc. Int. Conf. Sys. Engineering, Coventry, UK, 6-8 Sept., pp. 200-207, 2016.

[14] Belew, R. K. and Booker, L. B.: Proceedings of the Fourth International Conference on Genetic Algorithms, San Mateo, California, USA: MorGenetic algorithmn Kaufmann Publishers, 2015. 